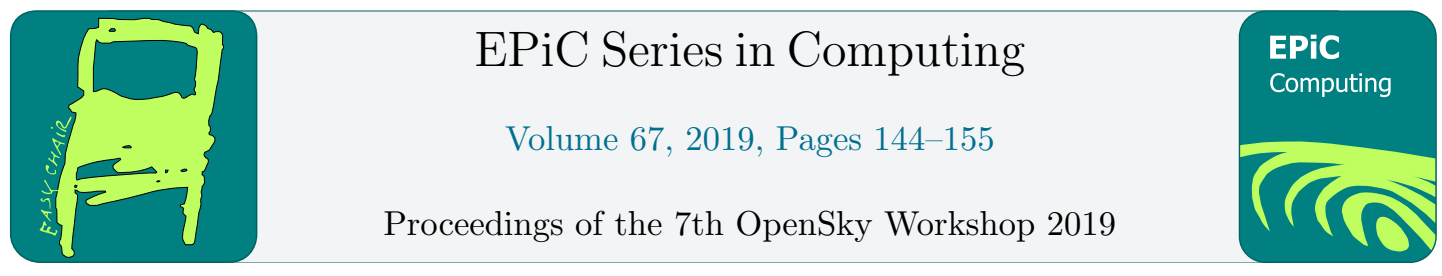

\title{
Regulation-Oriented Filtering in Web-Based Air Traffic Exploration
}

\author{
Philipp Meschenmoser, Juri Buchmüller, and Daniel A. Keim \\ University of Konstanz, \\ \{philipp.meschenmoser|juri.buchmueller|keim\}@uni-konstanz.de
}

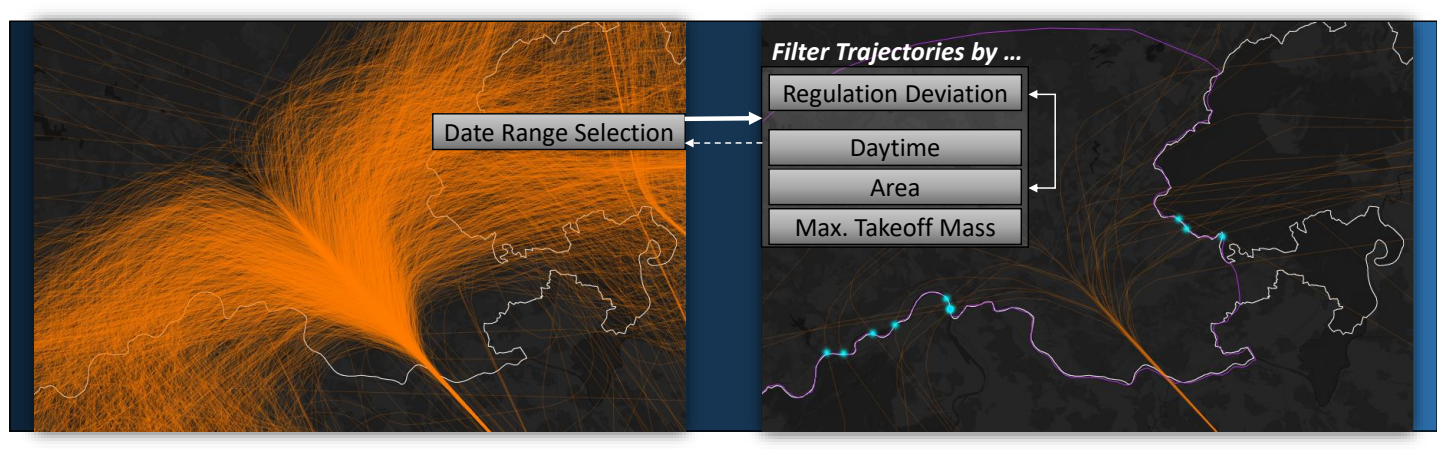

Figure 1: Left: Trajectories of 3,930 flights approaching the Zurich International Airport within two weeks in February 2019. Right: Identical date range, but filtered according to a specific scenario given by our regulation-oriented filtering scheme. Shown are trajectories of 32 flights that deviated from a night flight regulation during morning times and thereby crossed the purple area. Cyan dots indicate for each trajectory where the first deviation occurred. Considered trajectory data stems from the OpenSky Network [13].

\begin{abstract}
Airspace route planning relies on many regulations and individual factors that can be hard to understand for audiences without advanced domain knowledge. This aspect is problematic if regulations are discussed in complex debates about changing air traffic distributions, affecting the broad public in negative and positive ways. To increase accessibility and transparency, we propose a regulation-oriented scheme of trajectory filters that includes a fully automated detection component for regulation deviations. The scheme further includes filters by daytime, custom areas, MTOM, and is part of a client-independent web prototype. In this publication, we specify details on individual filters and their interplay $\left(1^{\text {st }}\right.$ contribution $)$, while putting a particular emphasis on the deviation detector $\left(2^{\text {nd }}\right.$ contribution).
\end{abstract}

C. Pöpper and M. Strohmeier (eds.), OpenSky19 (EPiC Series in Computing, vol. 67), pp. 144-155 


\section{Introduction}

Since the governing factors for air traffic can be manifold and hard to understand for specific cases, we present a regulation-oriented filtering scheme helping users to check compliance with and deviations from air space regulations. Air traffic is subject to multiple, partially overlapping sets of regulations, including general aviation safety rules, airspace-specific regulations, instructions of air traffic operators as well as the pilot's own judgment in the very end. Especially for general aviation, routing decisions follow further, intransparent decision factors such as optimized use of airspace, prioritization by importance, and economic or political provisions. Thus, understanding all contributing factors of air traffic routing for individual trajectories is extremely challenging, especially for interested audiences who are not familiar with all regulations in place.

With the scheme presented in this publication, we enable users to probe individual parts of regulations in place and to further explore details. Accordingly, given large amounts of trajectory data, users can effortlessly identify trajectories matching to very specific regulationrelated scenarios.

Especially in the vicinities of airports, many governing regulations and institutions exert influence on air traffic (re-)distribution: to ensure safety criteria, optimize traffic flow or minimize noise impact on the population living close to approach paths. Particularly when it comes to noise and health impacts due to aircraft noise, local conflicts arise quickly about the distribution and restriction of air traffic. We demonstrate our approach in Section 4 at the example of a use case showing the situation around Zurich International Airport (ZRH) in Switzerland, close to the German border. A cross-border conflict has led the German Federal Government to unilaterally impose regulations (e.g., concerning night approaches over Germany), which are compiled by the 220 th executive order to air traffic regulations (DVO-220, [11]). With our filtering scheme described in Section 3, we enable users to understand the compliance of air traffic with such regulations and find times and locations with behavior of interest. As well, we validate the detected deviations by comparison to public official records provided by the Zurich Airport AG.

\section{Related Work}

The analysis of air traffic has a pronounced history with roots in spatiotemporal data visualization and geosciences. While the focus of most related approaches clearly lies on enabling domain expert use cases, less attention has been paid to general audience solutions to explain complex air traffic situations with multiple and partially colliding governing factors and regulations. Several approaches exist to infer conclusions about individual aircraft trajectories and air traffic in general automatically, for example by the integration of heterogeneous data sources as shown by Strohmeier et al. [15] or the application of machine learning algorithms to identify outlier trajectories as presented by Li et al. [9].

Automatic approaches are well suited for large-scale explanation and outlier detection, yet are of limited practicality when it comes to the analysis of local areas with changing regional regulatory aspects and region-specific geographic, political, and societal contexts as in our use cases. Here, visual-interactive solutions provide both a spatial context and the ability to refine queries based on the results, context, and domain knowledge of the user. While a lot of conceptual groundwork is established (e.g., for trajectory comparison [6] or interactive filtering strategies [5] by Andrienko et al.), many works on interactive approaches focus on very specific expert tasks such as the identification of specific aircraft behaviour and decision 
making strategies (Andrienko et al. [4]), or on other movement properties such as traffic flows (Scheepens et al. [14]).

The most notable related approaches are provided by Hurter et al. [8], who present a flexible interaction framework for the spatial, temporal, and feature-based filtering of air traffic trajectories; and Buchmüller et al., who present a visual exploration and prediction system with fixed rule set implementation. While the former provides no possibility to encode contextual and domain knowledge about regulations, the latter does not allow the interactive and arbitrary combination of filters and statistical evaluation. The presented filtering scheme aims to provide a starting point to fill these gaps and allow for an easily accessible modeling and checking of regulations in the context of air traffic.

\section{$3 \quad$ Filtering Scheme}

For visualizing highly specific traffic scenarios with potential regulation deviations, we propose an easy-to-use, but still powerful scheme of trajectory filters. We demonstrate the scheme by flights arriving at ZRH. To keep our approach generic, we mainly assume aircraft trajectories with $t_{i}=$ (timestamp, latitude, longitude, altitude) tuples, stemming from any data source (e.g., OpenSky Network [13], FlightRadar24). In the following, we assume that $t_{i}$ are sorted by timestamps in ascending order. Section 3.1 shortly depicts the scheme overview and our filters' arrangement into the intended workflow. Sections 3.2 to 3.5 focus individual filter components and include both their relevance and specification.

\subsection{Overview and Workflow}

Our regulation-oriented scheme encompasses filters by, e.g., regulation deviations, overflown areas, daytimes, or maximum takeoff mass. The filters return matching trajectories and can be dynamically (de-)activated. Multiple active filters are inherently linked by a logical AND. See also Figure 3.

We implement the filtering scheme as part of a visual-interactive web prototype that provides rich means for exploration and analysis. Figure 2 illustrates the intended workflow of how the prototype's user optimally interacts with the filtering scheme.

\begin{tabular}{|c|c|c|}
\hline High-Level Filtering: & Interactive Sensitivity Refinement & Low-Level Filtering: \\
\hline 1) Date Range Selection \& Exploration & 2) Automated Deviation Detection & 3) Details Inspection \\
\hline
\end{tabular}

Figure 2: Intended interaction workflow with the proposed filtering scheme. Analyzing longterm developments of custom scenarios is doable at every stage.

(1) Initially, the user visits our web prototype, selects a date range of interest, and can visually explore all trajectories with an intersecting date range (i.e., the root set). The user can already exclude overall flight patterns and flows that appear irrelevant. (2) Having the root set reduced, the user now filters by trajectories that deviate from predefined regulations: e.g., night flight regulations. While the actual deviation detector is fully automated, the user can iteratively refine its sensitivity, i.e., filter already detected deviations by custom tolerances. (3) Having trajectories of deviating flights, the user can go further and use our filters to inspect details of even more individual scenarios. Additionally, the user can analyze long-term 
developments of the explored scenarios. With insights gained, the user can dynamically go back to previous steps to analyze (different) date ranges by further regulation-oriented scenarios.

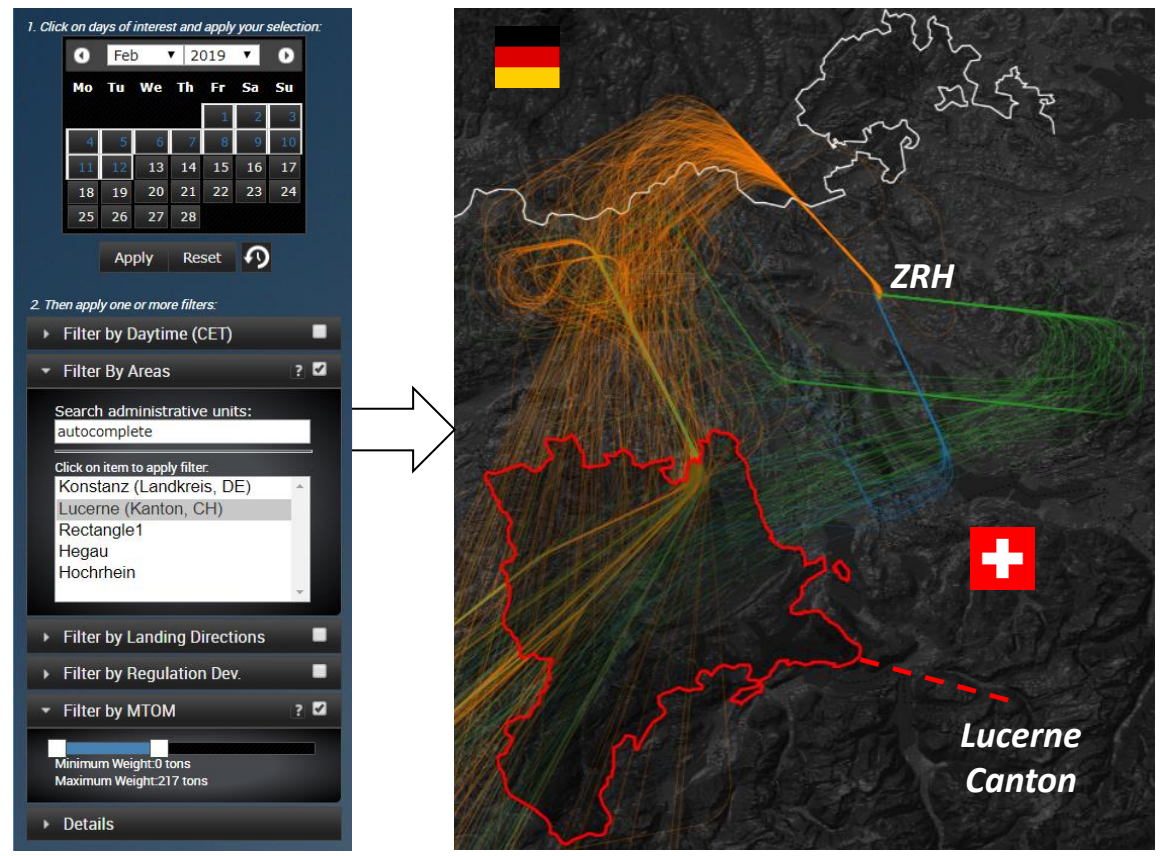

Figure 3: Left: Implementation of our scheme, showing an active area and MTOM filter. Right: The filtering result, i.e., 279 flights (of matching MTOM) that intersected Lucerne during 12 selected days. Trajectory data stems from the OpenSky Network [13].

This section continues with the automated deviation filter and detector (Section 3.2), area filter (Section 3.3), daytime filter (Section 3.4), and maximum takeoff mass filter (Section 3.5).

\subsection{Regulation Deviation Filter and Detector}

With this filter, the user reduces the current trajectory set to these trajectories that (additionally) deviate from at least one predefined regulation. Here four DVO-220 regulations (two for night flights, two for minimum sector altitudes) can be dynamically selected through checkboxes, triggering fast attribute checks in a backend database.

This filter relies on a fully automated deviation detector that assumes regulations to be defined as 8-tuples (area, dow, holidays, approaching, runways, $h_{\text {start }}, d, p$ ). area gives the regulation-relevant multipolygon, dow $\subseteq[0,6]$ gives the regulation-relevant days of the week that can be enriched by public holidays $\in\{$ true,false $\}$. With approaching $\in\{$ true,false $\}$ and the number set runways, the regulation's day-wise time window is given by the start daytime $h_{\text {start }}$ and window duration $d$. Lastly, the tuple $p$ specifies further parameters, such as minimum flight altitude. As an example, (area, $\{0,6\}$, true, true, $\{14,16\}, 00: 00,09: 00,()$ ) covers one part of the extended night flight regulation over Germany on weekends and holidays. See Table 1 for further details. The detector automatically translates such 8-tuples to a database query and finally stores the responsible trajectory points of deviating flights. Accordingly the detector has to be active only once, thus ensuring precalculations and efficiency. 


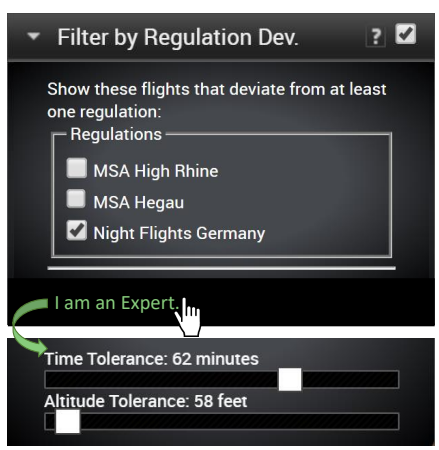

Figure 4: Filter trajectories by regulation deviations and, as part of a light-weight expert mode, specify the detection sensitivity.

Further, the foregoing tuple formalization (a) enables the administrator to effortlessly add and modify regulations, and (b) is the basis for a user-steered function to control the detector sensitivity. In the following, we motivate the latter function and describe our realization.

Often, regulation deviations mirror edge cases, e.g., when long-distance flights have delayed approaches and thereby deviate from a night flight regulation by only a few minutes, cf. [10]. Clearly, the public perceives such minor deviations less explicitly, than night flight deviations that occur by the middle of the night and, in the long term, can increase the likelihood of health issues. Furthermore, differentiating between deviation strengths is important to reduce the airport's investigative efforts.

Consequently, as part of a light-weight expert mode, the user of our prototype can specify the detection sensitivity by defining time and altitude tolerances. Using the interactive sliders in Figure 4, the user can analyze relatively strong deviations from the night flight regulation. Thus she/he dismisses flights where all deviations occur within the first/last 62 minutes (max. 90 minutes) of the regulation-relevant time frame. Specifying the altitude tolerance (max. $1000 \mathrm{ft}$ ) works analogously.

Specifying the detector sensitivity is again efficient, as we store for each deviating trajectory point $t[i]$ three measures of deviation strength: (a) minutes after the regulation time frame's beginning, (b) respectively before its ending, and (c) the altitude difference from the regulation's minimum altitude. Thus sliding interaction relies on a simple scan over these $t[i]$, and once a single tuple $t[j]$ with threshold-exceeding deviation strengths is found, the according trajectory can be returned. Exploiting properties of the strength measures can imply further optimizations: (a) is strictly monotonically increasing, analogous for (b), and (c) is usually monotonically decreasing in approach scenarios.

The following filters allow to further refine the obtained set of deviating trajectories, but also to construct own regulation-related scenarios more dynamically.

\subsection{Area Filter}

With this filter, the user selects trajectories that (additionally) intersect at least one of a set of suggested or customly defined areas. The suggested areas, given by an autocomplete search, encompass approach areas as defined by DVO-220 regulations, as well as German and Swiss administrative areas that cover the scopes of interest of, e.g., local citizens' groups, political institutions, and economic organizations. Thus we provide 638 areas that include German counties ('Landkreise'), governorates ('Regierungsbezirke'), states ('Bundesländer'), next to Swiss districts ('Bezirke') and cantons. Filtering by suggested areas can come with another benefit. By selecting a relatively small area (e.g., the Berlin county), almost only flights that arrive/depart at Berlin's TXL or SFX are returned, thus our filter can be a substitute for a separate filter by airport. Lastly, note that filtering by suggested areas is very fast, as intersections can be easily precalculated.

Additionally, the user can define custom areas by interactively drawing circles (cf. holding stacks, and point-based noise models assuming spherical sound waves), rectangles (cf. straightline segments, and line-based noise models [12]), as well as arbitrary simple polygons (that need more drawing effort but allow even more customization). 


\subsection{Daytime Filter}

Given an active area filter, the daytime filter returns these trajectories that (additionally) intersect the specified area(s) during the daytime interval $\left(h_{\text {start }}, h_{\text {end }}\right)$, CET. Without an active area filter, the daytime filter returns these trajectories that are during $\left(h_{\text {start }}, h_{\mathrm{end}}\right)$, CET, in the vicinity to $\mathrm{ZRH}$. Presumably, UTC might be preferred over a local time zone at analyses covering multiple time zones.

The user specifies $\left(h_{\text {start }}, h_{\text {end }}\right)$ by drag handlers in a custom clock widget, see Figure 5. Compared to alternatives (e.g., range sliders, drop-menus), this clock widget entails two important benefits.

First, the widget realizes a metaphor for a well-known real-world object. Second, the widget allows to easily specify time windows that cover the intra-day limit $\left(h_{\text {start }}>h_{\text {end }}\right)$ : a feature that is highly relevant for analyzing flight movements during night times (cf. [11, 2]). Note that, with $h_{\text {start }}>h_{\text {end }}$, it can be necessary to load trajectories that were not part of the initial root set.

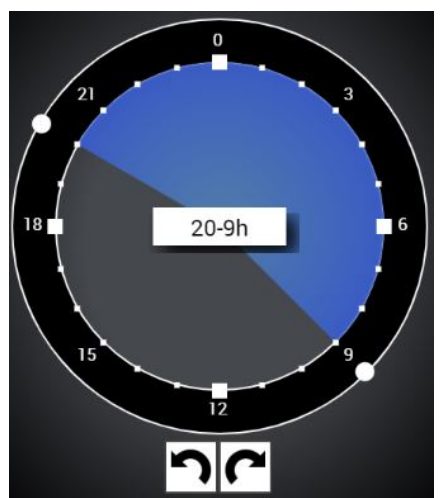

Figure 5: Clock Widget. The range $8 \mathrm{pm}$ to $9 \mathrm{am}$ is selected after having dragged the white circles. Arrow buttons for iterating.

\subsection{Maximum Takeoff Mass (MTOM) Filter}

This filter returns these trajectories whose aircraft (additionally) have a MTOM in $\left[\right.$ mtom $_{\min }$, mtom $\left._{\max }\right]$. This filter is relevant for multiple reasons. E.g., routes for heavy aircraft are often determined by special regulations, and the airlines' landing charges are higher for heavier aircraft. In particular, a higher MTOM often implies higher emissions (e.g., noise), so that regulation deviations might be even more perceivable on the ground. Note that, with the MTOM being constant along a trajectory, this filter enables an intuitive interplay with previous filters and is representative for further constant attributes (e.g., noise profiles, operating airlines).

\section{Case Study: Deviations while approaching $\mathrm{ZRH}$}

We showcase our deviation detector and filtering scheme at regulations concerning minimum sector altitudes (MSAs, definition in Section 4.1) and night approaches to ZRH. Considering the 8-tuple formalization from Section 3.2, the implementation of a subset of DVO-220 regulations is given by Table 1. In Section 4.1, we depict our detector results for two MSAs (i.e., 1) and 2) in Table 1), Section 4.2 refers to detector results for night approaches and entails a comparison with ground truth data from the Zurich Airport AG.

We emphasize that, with our scheme and case study, it is not our objective to adopt party in the on-going debates about route redistributions. Instead, while using publicly available data and exemplary regulations, we present an easily accessible information medium that shall increase transparency for several interest groups and could even stimulate further discussions.

In the following, trajectories will be considered that result from ADS-B broadcasts and are gathered by the OpenSky Network [13]. At MSA deviations we refer to the more accurate 
8-tuple formalization

\begin{tabular}{|c|c|c|c|c|c|c|c|c|c|c|}
\hline Regulation & Ref. to DVO-220 & Description & Area & Dow & Holidays & Approaching & Runways & $h_{\text {start }}$ & Duration (hh:mm) & $\mathrm{p}$ \\
\hline 1) MSA High Rhine & \begin{tabular}{|c|} 
6th Adjustment, \\
$\$ 2,(4)$
\end{tabular} & $\begin{array}{l}\text { Approaching flights with } \\
\text { anytime MSA of } 5700 \mathrm{ft} \text {. }\end{array}$ & High Rhine, see ref. & {$[0,6]$} & True & True & * & $00: 00$ & $24: 00$ & $\begin{array}{c}\text { Minimum Altitude } \\
=5700 \mathrm{ft}\end{array}$ \\
\hline 2) MSA Hegau & $\begin{array}{c}\text { 6th Adjustment, } \\
\$ 2,(4)\end{array}$ & $\begin{array}{l}\text { Approaching flights with } \\
\text { anytime MSA of } 4500 \mathrm{ft} \text {. }\end{array}$ & Hegau, see ref. & {$[0,6]$} & True & True & * & $00: 00$ & $24: 00$ & $\begin{array}{c}\begin{array}{c}\text { Minimum Altitude } \\
=4500 \mathrm{ft}\end{array} \\
\end{array}$ \\
\hline $\begin{array}{l}\text { 3) Night Flights } \\
\text { Workdays } 1\end{array}$ & $\$ 2,(8)$ & $\begin{array}{c}\text { Approaching night flights to } \\
\text { runway } 14 \text { or } 16, \text { from Monday } \\
\text { to Friday (incl.). Morning } \\
\text { hours. }\end{array}$ & $\begin{array}{l}\text { High Rhine, Hegau, } \\
\text { RILAX Corridor }\end{array}$ & {$[1,5]$} & False & True & $\begin{array}{l}\{14,16\} \\
\text { (North) }\end{array}$ & $00: 00$ & 07:00 & - \\
\hline $\begin{array}{l}\text { 4) Night Flights } \\
\text { Workdays } 2\end{array}$ & $\$ 2,(8)$ & "“". Evening hours. & $\begin{array}{l}\text { High Rhine, Hegau, } \\
\text { RILAX Corridor }\end{array}$ & {$[1,5]$} & False & True & $\begin{array}{l}\{14,16\} \\
\text { (North) }\end{array}$ & 21:00 & 03:00 & - \\
\hline $\begin{array}{c}\text { 5) Night Flights } \\
\text { Weekend \& Holidays } \\
2\end{array}$ & $\S 2,(8)$ & \begin{tabular}{|c|} 
Approaching night flights to \\
runway 14 or 16 , on \\
Saturdays, Sundays, and public \\
holidays in Baden- \\
Wuerttemberg. Morning \\
hours.
\end{tabular} & $\begin{array}{l}\text { High Rhine, Hegau, } \\
\text { RILAX Corridor }\end{array}$ & $\{6,0\}$ & True & True & $\begin{array}{l}\{14,16\} \\
\text { (North) }\end{array}$ & $00: 00$ & 09:00 & - \\
\hline $\begin{array}{c}\text { 6) Night Flights } \\
\text { Weekends/ Holidays } \\
1\end{array}$ & $\S 2,(8)$ & "“. Evening hours. & $\begin{array}{l}\text { High Rhine, Hegau, } \\
\text { RILAX Corridor }\end{array}$ & $\{6,0\}$ & True & True & $\begin{array}{l}\{14,16\} \\
\text { (North) }\end{array}$ & $20: 00$ & 04:00 & \\
\hline
\end{tabular}

Table 1: DVO-220 regulations (for minimum sector altitudes and night approaches over Germany) that we implement as part of the fully automated deviation detector. Here, the original night flight regulations are internally distinguished by evening and morning hours to better handle cases such as the morning approaches after public holidays. Still, the user obtains a simplified view on only three aggregated regulations.

geometric GPS altitudes above mean sea level. Trajectory tuples are interpolated to a time resolution of ten seconds, which is reasonable in particular at approaching, thus slowing down flights.

\subsection{Minimum Sector Altitudes}

MSAs "guarantee 1,000 feet of terrain clearance within 25 nautical miles" ([7], p. 120). Thus MSAs are in particular relevant at adverse weather conditions, at a pilot's self-reliant navigation, or when air traffic controllers have to handle an aircraft apart from predefined routes. §2, (4) of the $6^{\text {th }}$ DVO-220 adjustment (17 ${ }^{\text {th }}$ August 2015, cf. [11]) defines these altitudes for two areas in Germany. We denote the areas as High Rhine (MSA of 5,700 ft AMSL) and Hegau (MSA of 4,500 ft AMSL). See also Figure 6.

At first, we regard deviations from the Hegau MSA of 4,500 ft AMSL. Figure 7 shows different approach scenarios where flights crossed the Hegau area during the first week of August 2019. Within this week, 14 approaches flew below the MSA, corresponding to $\approx 0.58 \%$ of all approaches. 800 approaches deviated from the MSA between $1^{\text {st }}$ January and $31^{\text {st }}$ October 2019 (293 days; $\approx 0.82 \%$ of all approaches). Partly these deviations stem from light-weight aircraft that often fly on low altitudes due to technical constraints (e.g., no pressurized cabin). Another deviation reason will be now illustrated by referring to the High Rhine MSA.

Considering the High Rhine MSA of 5,700 ft AMSL, Figure 8 encodes trajectories for 5,403 flights that flew below the MSA between the $1^{\text {st }}$ and $21^{\text {st }}$ August 2019. The overlaid density map illustrates for each trajectory where the first deviation occurred. The flights mainly represent north landings to runway 14 or 16 that often have to sink below the MSA on German territory, even before the final approach. The denser areas (A) and (B) in Figure 8 represent first deviations from flights that entered Germany already under 5,700 ft AMSL. These flights mainly came from NEGRA, respective GIPOL, and sank below the MSA during their left-, respective right-curve, before flying on the straight-line, final approach. First deviations between (A) and (B) are relatively scattered in space. (C) indicates an extremely dense area placed on the 


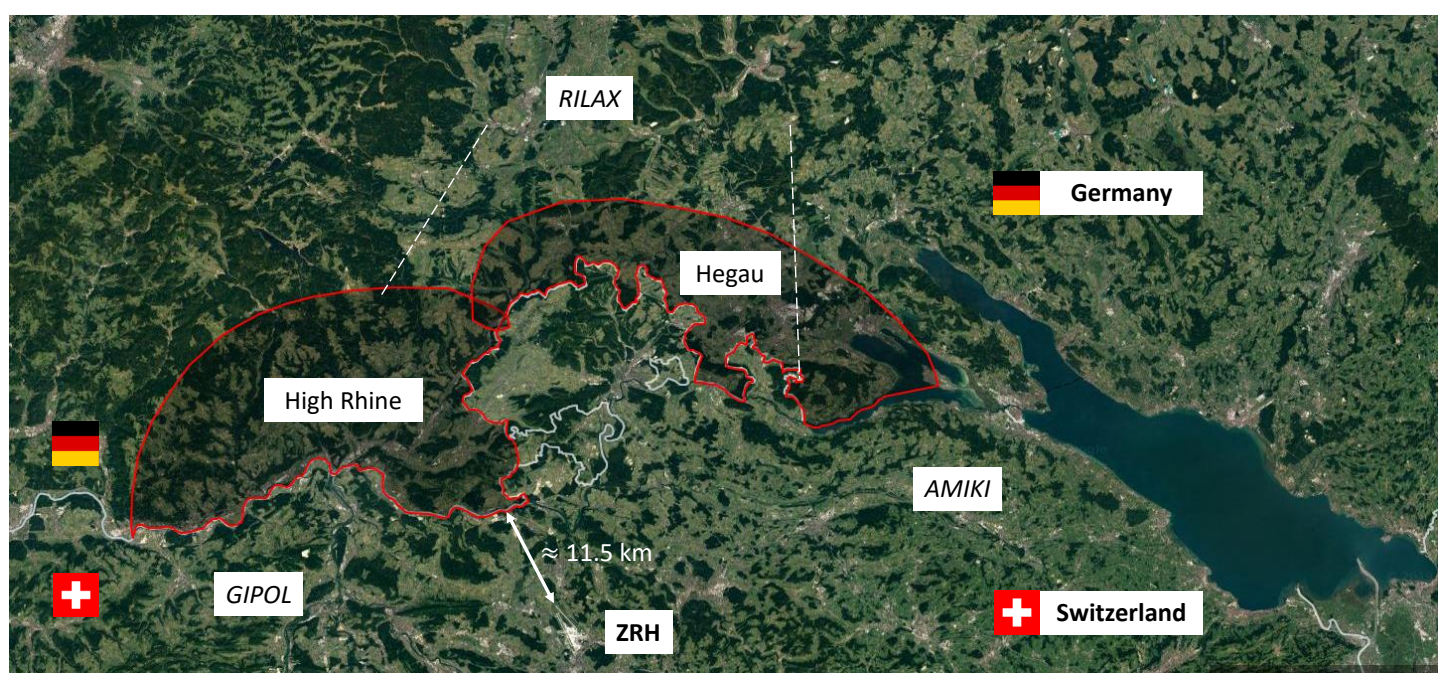

Figure 6: The High Rhine and Hegau area are considered for minimum sector altitudes. RILAX, GIPOL, and AMIKI are waypoints that are often passed by before north landings to runway 14 or 16. The white dashed lines indicate a common flight corridor after having passed RILAX.
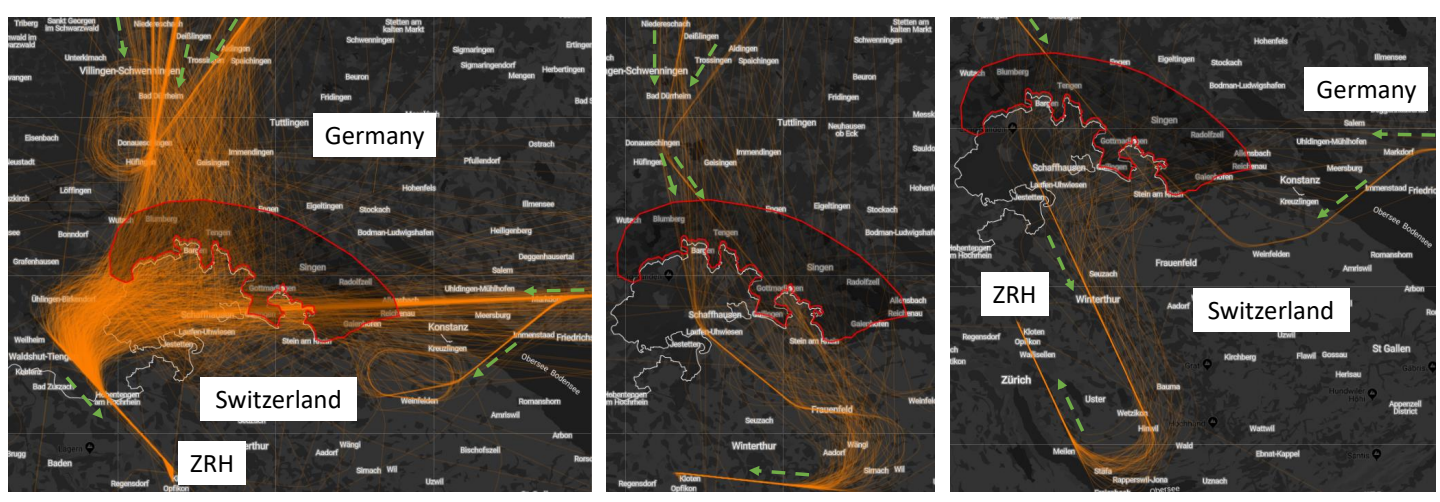

Figure 7: Flights to ZRH that pass by the Hegau area (red), $1^{\text {st }}$ to $7^{\text {th }}$ August 2019, 2428 approaches in total. Left: 872 north landings. Middle: 98 east landings. Right: 59 south landings. Trajectory data from the OpenSky Network [13].

final approach segment: 293 first deviations occurred solely within the black square's area (side length $=750 \mathrm{~m}$ ). The dense area around $(\mathrm{C})$ begins already $\approx 10.75 \mathrm{~km}$ before crossing the German-Swiss border.

With OpenSky Network data, the 5,403 deviating flights represent $\approx 73.81 \%$ of the total number of ZRH approaching flights between the $1^{\text {st }}$ and $21^{\text {st }}$ August 2019. Regarding the range between $1^{\text {st }}$ January and $31^{\text {st }}$ October 2019, our detector identifies 67,150 deviating approaches, which corresponds (analogously to before) to $\approx 68.86 \%$.

We interviewed air traffic controllers as well as pilots for passenger planes and asked them to further explain these multitudes of MSA deviations. According to them, sinking below the 5,700 ft AMSL distinctly before the German-Swiss border is an operational and technical 
necessity when landing on runway 14 or 16. Further, pilots may sink below the MSAs during final approaches or if they follow a route with another defined minimum altitude. Thus our results show (1) an example of how regulations can be overlapping, even conflicting, and (2) how visual-interactive methods can help in improving the understanding.

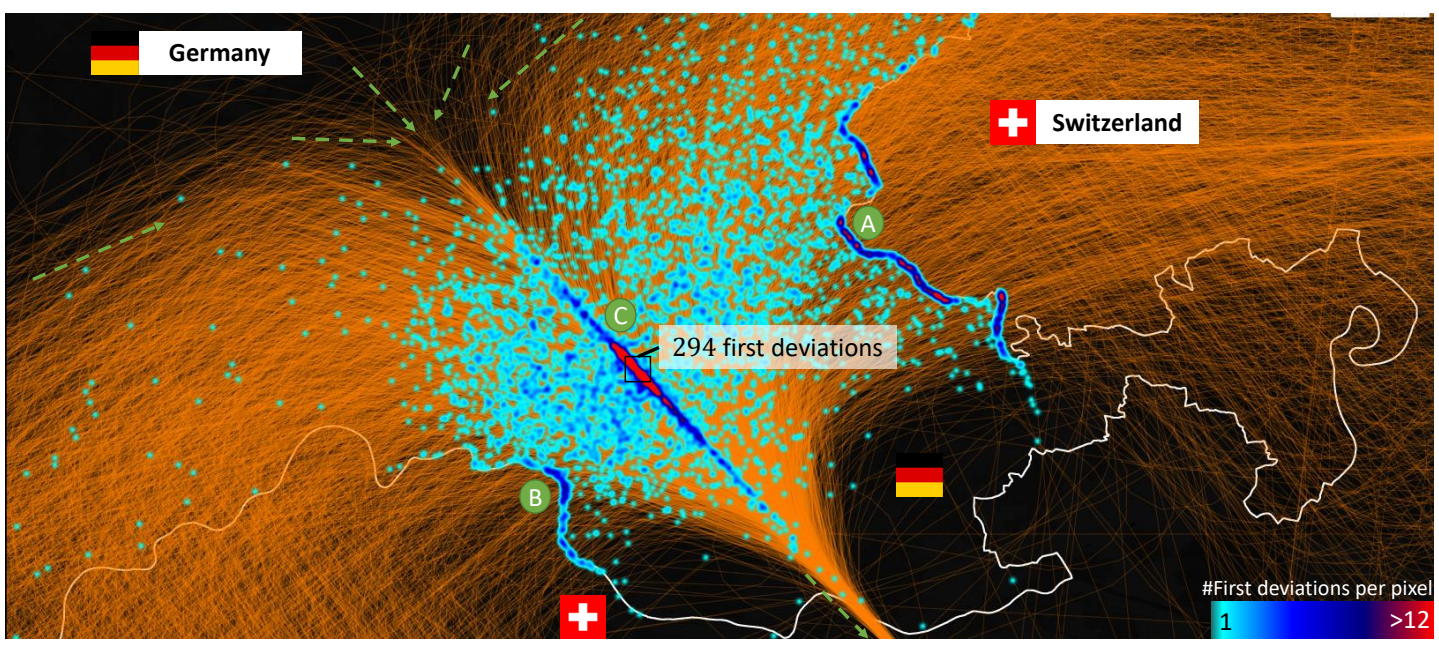

Figure 8: Density map (max. intensity per pixel: 13) showing for each trajectory the first deviation from the High Rhine MSA for three weeks in August 2019. The German-Swiss border is given by the mostly longitudinal white line. Green arrows indicate the overall trajectory flow. Trajectory data from the OpenSky Network [13]. Note that we consider geometric altitude. First deviations at barometric altitude would occur in the present case even earlier but the overall distribution would remain similar.

\subsection{Night Approaches over Germany}

According to the DVO-220 [11, 3], certain approach scenarios shall be performed over German territory (a) on workdays only between 7 am and $9 \mathrm{pm}$, and (b) on weekends and public holidays in Baden-Wuerttemberg only between 9 am and $8 \mathrm{pm}$. In the following we focus north landings again, as defined in, e.g., the DVO-220 [11] and its recent adjustments, §2, (5) 1.-6. and (7) 1.-2. At north landings, flights mainly come from either Swiss waypoints (GIPOL, AMIKI) or German waypoints (RILAX, NEGRA), enter the High Rhine area (as in Figure 8) and land on runway 14, respective 16. Consequently for detecting deviations, we use the High Rhine and Hegau area, as well as the corridor between these areas and RILAX. See also the formalizations 3) to 6) in Table 1.

Now we concentrate on these days from $1^{\text {st }}$ January to $31^{\text {st }}$ October 2019 for which the automated detector returns at least eight deviating night approaches. The latter threshold choice is reasonable as an interactive time series chart in our prototype clearly brings out the cutting line between days with common and anomalously high counts of deviating flights.

For the resulting 31 days, the blue bars in Figure 10 encode the counts of deviating flights. With OpenSky Network data, our detector returns 911 deviating flights during these 31 days: This corresponds to $\approx 11.24 \%$ of all north landings and $\approx 8.91 \%$ of all approaches. We can use the daytime filter (Section 3.4) to distinguish between deviations during morning and evening hours. 652 out of 911 flights $(=73.87 \%)$ cause deviations during morning hours: i.e., from 2 
am to $9 \mathrm{am}$; the remaining percentage reflects $8 \mathrm{pm}$ to $2 \mathrm{am}$. This ratio between morning and evening hours is in the same magnitude as published ratios, cf. [1].

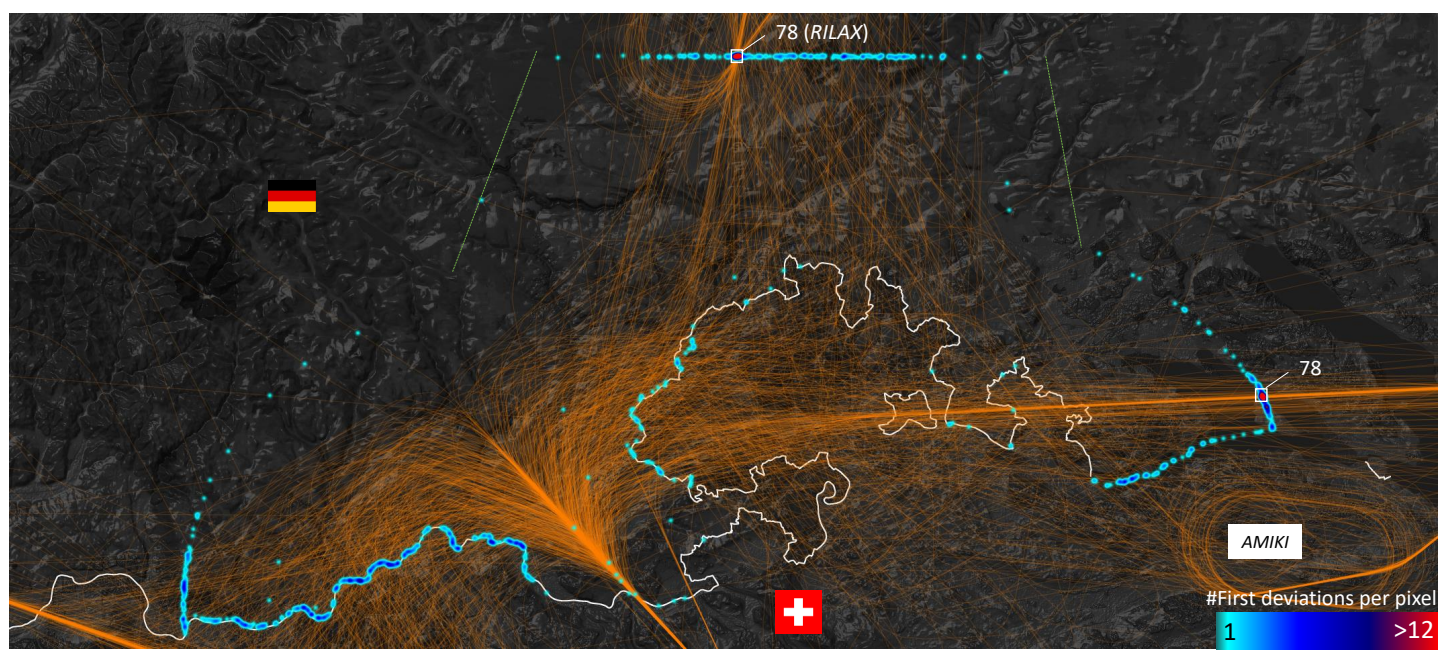

Figure 9: Trajectories for 911 deviating night approaches during the 31 days with at least eight deviating flights. Again, the density map (max. intensity per pixel: 13) encodes only first deviations. Trajectory data from the OpenSky Network [13].

Again, the density map in Figure 9 encodes for each trajectory where the first deviation occurred. The spatial distribution of deviations clearly reflects the considered areas' boundaries. Thus it is very likely that flights crossed Germany during night times even before entering these areas. Similar to Figure 8, there are two areas of very high deviation density: at the RILAX waypoint and the entry point to the Hegau area after passing by NEGRA. In both cases, there are 78 first deviations inside squares of side length $750 \mathrm{~m}$. Very few first deviations are located more to the inside of the checked areas. These deviations refer to flights that entered the areas shortly before 8 (or 9) pm but could not leave them in time. By decreasing the detector sensitivity, the first deviations of evening flights will move in general to the final approach segment and German-Swiss border. Vice versa for morning flights. At some point of lowered sensitivity, there would not be any first deviation and the according trajectory becomes hidden.

As a next step, we can partly evaluate our detector's performance by ground truth data that the Zurich Airport AG provides. Once in a month, the AG publishes the day-wise counts of flights [1] that cause justified exceptions from night flight regulations in the DVO-220 (e.g., due to emergencies or adverse weather conditions). Clearly analyzing deviations and justifying them comes with much administrative effort. However, these steps are necessary because deviations without plausible justification can even lead to legal consequences. Now we regard only days between $1^{\text {st }}$ January and $31^{\text {st }}$ October 2019 for which the Zurich Airport AG reports at least eight justified exceptions. The other days, with fewer exceptions, are relatively rare and usually based on one or two deviating flights.

The official exception counts and justifications from the Zurich Airport AG are encoded by the orange bars in Figure 10. Consulting our detector results again, there is a very high overlap of days: The detector correctly retrieves 28 out of 29 peak days that the Zurich Airport AG reports. For the not retrieved day (19 ${ }^{\text {th }}$ March 2019) the Zurich Airport AG reports 18 justified exceptions. Vice versa, our detector returns three additional days for which the AG reports 
zero justified exceptions. One of these days is Whitmonday (10 ${ }^{\text {th }}$ June 2019$)$ that should be covered by extended night flight regulations [11]. Interestingly, on Ascension Day (30 ${ }^{\text {th }}$ May 2019) there is a day-wise overlap again. In general, next to the high day overlap, also the actual deviation (or exception) counts per day are in very similar magnitudes. At 22 days, our detector returns deviation counts that correspond to $90-110 \%$ of the justified exception counts. We explain differences from the optimal measure value $100 \%$ primarily as (1) data can be incomplete, (2) we do not implement the entire DVO-220, and (3) not each deviation becomes a justified exception.

By comparing our results with the aggregated ground truth, we can conclude that our detector returns deviations with adequate confidence. Additionally, our detector consumes here publicly available data and works fully automated in near realtime. In contrast, to get the official counts of justified exceptions for a recent day, one would have to wait, in the worstcase, longer than one month for the public release (or try a custom request). Moreover, our prototype's user benefits from visual-interactive methods at analyzing results in a very detailed and transparent manner.

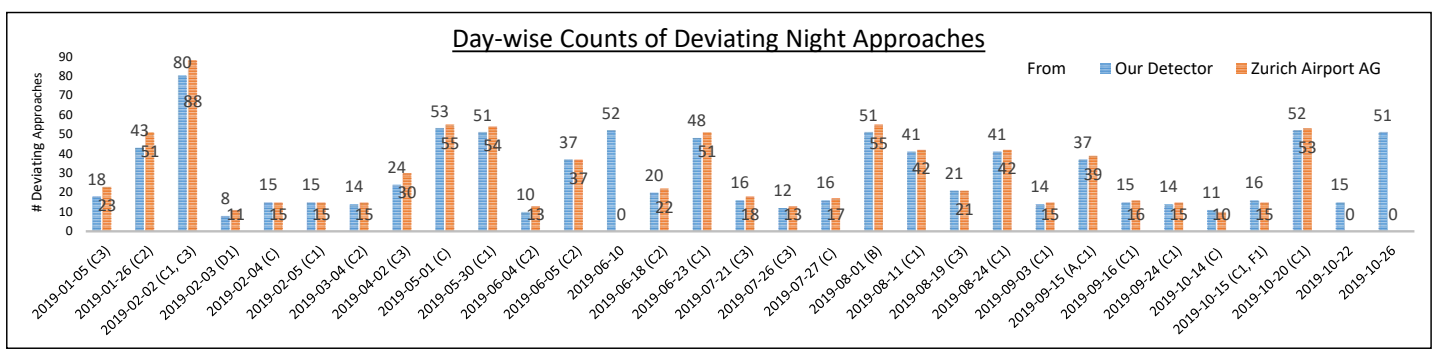

Figure 10: Day-wise counts of deviating night approaches from our detector (blue bars), next to official numbers and justifications for regulation exceptions provided by the Zurich Airport AG (orange bars, [1]). These justifications are encoded by tokens next to the date labels. A: Emergency. B: Security reason. C: Altitude or sight below minimum. C1: Short sight. C2: Tailwinds. C3: Adverse weather conditions. D1: Snow services. F1: Ambulance flight.

\section{Conclusion \& Future Plans}

For exploring air traffic, we present an easy-to-use, but still powerful, regulation-oriented filtering scheme having an automated deviation detector as its core. With our web-based implementation, the scheme shall make the multitude of regulations and their compliance more transparent and accessible for less familiar audiences. Regarding future plans, we envision the scheme to be part of a web platform constituted by even more intelligent and automatized analysis procedures. While regulations will become more complex, we are looking forward to automatically derive reasons for deviations, and evaluate uncertainties of deviations and their reasons. Therefore we emphasize a visualization design to effectively and efficiently communicate results, aiming to support a broad audience at the understanding of complex air traffic regulations and their compliance. 


\section{References}

[1] Airport Zurich AG. Justified dvo-220 exceptions. https://www.flughafen-zuerich.ch/ / media/flughafenzh/dokumente/das_unternehmen/laerm_politik_und_umwelt/ausnahmen_von_ der_dvo_20191106.pdf. [Online; accessed 12-November-2019].

[2] Airport Zurich AG. Night flight regulations airport zurich. https://www.flughafen-zuerich.ch/ unternehmen/medien/aktuelle-themen/nachtflugsperre. [Online; accessed 29-October-2019].

[3] Airport Zurich AG. Overview dvo regulations. https://www.flughafen-zuerich. ch/ /media/flughafenzh/dokumente/das_unternehmen/laerm_politik_und_umwelt/ ueberblick-dvo-regelung_2018.pdf. [Online; accessed 04-November-2019].

[4] Gennady Andrienko, Natalia Andrienko, Georg Fuchs, and Jose Manuel Cordero Garcia. Clustering trajectories by relevant parts for air traffic analysis. IEEE transactions on visualization and computer graphics, 24(1):34-44, 2017.

[5] Natalia Andrienko, Gennady Andrienko, Elena Camossi, Christophe Claramunt, Jose Manuel Cordero Garcia, Georg Fuchs, Melita Hadzagic, Anne-Laure Jousselme, Cyril Ray, David Scarlatti, et al. Visual exploration of movement and event data with interactive time masks. Visual Informatics, 1(1):25-39, 2017.

[6] Natalia Andrienko, Gennady Andrienko, Jose Manuel Cordero Garcia, and David Scarlatti. Analysis of flight variability: a systematic approach. IEEE transactions on visualization and computer graphics, 25(1):54-64, 2018.

[7] D.J. Clausing. The Aviator's Guide to Navigation. McGraw-Hill Education, 2007.

[8] Christophe Hurter, Benjamin Tissoires, and Stéphane Conversy. Fromdady: Spreading aircraft trajectories across views to support iterative queries. IEEE transactions on visualization and computer graphics, 15(6):1017-1024, 2009.

[9] Yue Li, Kaiquan Cai, and Su Yan. Critical flight trajectory identification via machine learning for large-scale trajectory management. In 2018 IEEE/AIAA 37th Digital Avionics Systems Conference (DASC), pages 1-7. IEEE, 2018.

[10] Eric Mueller and Gano Chatterji. Analysis of aircraft arrival and departure delay characteristics. In AIAA's Aircraft Technology, Integration, and Operations (ATIO) 2002 Technical Forum, page 5866, 2002.

[11] Federal Aviation Administration of Germany. Zweihundertzwanzigste durchführungsverordnung zur luftverkehrs-ordnung. https://web.archive.org/web/20130927084327/http://www . fluglaermschutz.ch/frameset/fakten-und-infos/220dvo.pdf, 2005. [Online; accessed 22-August-2019].

[12] Federal Environment Agency of Germany. Guidance for calculating noise abatement zones. https://www.hlnug.de/fileadmin/dokumente/laerm/gesetze/flugverkehr/AzB_07.pdf, 2007. [Online; accessed 23-August-2019].

[13] Matthias Schäfer, Martin Strohmeier, Vincent Lenders, Ivan Martinovic, and Matthias Wilhelm. Bringing up opensky: A large-scale ads-b sensor network for research. In Proceedings of the 13th international symposium on Information processing in sensor networks, pages 83-94. IEEE Press, 2014.

[14] Roeland Scheepens, Christophe Hurter, Huub Van De Wetering, and Jarke J Van Wijk. Visualization, selection, and analysis of traffic flows. IEEE transactions on visualization and computer graphics, 22(1):379-388, 2015.

[15] Martin Strohmeier, Matthew Smith, Vincent Lenders, and Ivan Martinovic. Classi-fly: Inferring aircraft categories from open data. arXiv preprint arXiv:1908.01061, 2019. 